\title{
The method of the galactic rotation distances
}

\author{
C. Jaschek, A. Valbousquet and F. Ochsenbein \\ Observatoire de Strasbourg, 11 rue de l'Université, 67000 Strasbourg, France
}

Received September 19; accepted December 1, 1995

\begin{abstract}
The method of galactic rotation distances is investigated by means of numerical simulations and found to work properly, even when the dispersion of the absolute magnitudes or the peculiar velocity dispersion are large. We apply the method to a large sample of more than 600 galactic supergiants and bright giants. We derive average mean absolute magnitudes which confirm the average absolute magnitudes found by either Blaauw or Schmidt-Kaler. Our calibration has the advantage to be based upon both a single method and a large number of stars. We show that the method works properly only with large samples. Therefore no subdivisions according to spectral types can be derived. We show further that the method is stable in the sense that small variations in the input parameters produce only small changes in the results.
\end{abstract}

Key words: stars distances — methods: numerical — methods: statistical — stars: fundamental parameters

\section{Introduction}

The method of galactic rotation distances assumes that the radial velocity of a given object is due to the sum of three components: the reflected solar motion, a peculiar component (i.e. peculiar to each star) and the reflected galactic rotation. Since in the galactic rotation formula enters the heliocentric distance to the object, if one knows all other parameters, one may obtain the distance. To eliminate the peculiar motion component, the method is applied to a large number of stars because in such a case the peculiar motion components may be assumed to cancel out. One obtains then the average distance of the group. Furthermore the method works best with objects at rather large heliocentric distances, because otherwise the galactic rotation component is small. If one uses stars with heliocentric distances less than one kpc, one can use the simplified rotation formula for the radial velocity:

$$
V(\operatorname{rot})=A \cdot r \sin 2 l \cdot \cos ^{2} b
$$

to which one may add a $K$ term. The reality of th $K$ term has been debated extensively in the literature, without a definitive conclusion (See for instance Kuijken \& Tremaine 1992). We shall keep it as an option.

The general expression for the radial velocity of a stars is thus:

$$
V(\mathrm{obs})=V_{0} \cos \lambda+A \cdot r \sin 2 l \cos ^{2} b+K+\varepsilon
$$

Send offprint requests to: C. Jaschek, Teso de la Feria 39, Salamanca, Espagne where $V$ (obs) is the observed radial velocity, $l$ and $b$ are the galactic longitude and latitude, $\lambda$ is the angular distance from the solar apex, and the peculiar velocity component. If one takes a large number of similar objects one can apply a least square procedure which permits then to obtain $V_{0}$, $K$ and the product $(A r)$, if one assumes furthermore that:

$$
\frac{1}{N} \Sigma \varepsilon_{i} \rightarrow 0
$$

a fact which, given the random nature of the peculiar velocities, seems acceptable.

The procedure is thus easy to apply, provided one has a sufficiently large group of similar stars with known radial velocity so as to make sure that condition (3) is fulfilled. The quantities $V_{0}, l_{0}$ and $b_{0}$, are either known or can be derived from the radial velocities. In the latter case one has to write the Eq. (1) in the following form

$$
\begin{aligned}
V(\mathrm{obs}) & =\varepsilon+V_{0} \sin b \cdot \sin b_{0}+V_{0} \cos b \cdot \cos b_{0} \cdot \sin l \cdot \sin l_{0}+ \\
& +V_{0} \cos b \cdot \cos b_{0} \cdot \cos l \cdot \cos l_{0}+A r \cdot \sin 2 l \cos 2 b+K
\end{aligned}
$$

and from there on one may determine the unknowns $V_{0} \sin b_{0}, V_{0} \cos b_{0} \sin l_{0}, V_{0} \cos b_{0} \cos l_{0}$ from which one can obtain in turn $V_{0}, l_{0}, b_{0}$.

What remains to be fixed is the rotation constant $\mathrm{A}$. This constant has been determined often in the literature; the IAU recommended value being $15 \mathrm{~km} / \mathrm{s} . \mathrm{kpc}$.

The most critical parts of the procedure are the "similarity" of the stars to which the method is applied and the definition of what means "a reasonably large number of objects". 
With regard to the first point, since one is interested in the average absolute magnitude, this means that one must choose a group of stars which are (presumably) homogeneous from this point of view. Usually this is done through a spectral classification procedure (for instance, using only stars of the same luminosity class) or with photometric indices (using only stars having closely similar Hbeta indices) or through the use of "similar" lightcurves (for instance when dealing with Cepheids).

With regard to the second point, that of "a reasonably large number of stars" one would say from statistical grounds that one thousand objects should be a right order of magnitude. However astronomers apply the method to small samples containing of the order of twenty or so stars. The reason for doing so is that usually no large number of objects is available. One must also add that if the number of stars in a sample is large, usually the homogeneity of the group becomes problematic. In order to clarify how the method works, we have done a number of numerical simulations.

\section{Test of the method}

For details of how to create an artificial sample of stars see the recent paper by Jaschek et al. (1994). We shall not repeat what was written in the paper, but simply specify the parameters we are using in the present work.

\subsection{Test of the method of galactic rotation distances}

We have simulated a sample of high luminosity stars with $M=-5.4$. To do so, we have assumed that the stars are distributed exponentially in height above the galactic plane with an exponentially decreasing density with $\beta=50 \mathrm{pc}$ and constant density in the galactic plane. The solar velocity was assumed to have components $V_{x}=11.4$ $V_{y}=14.6$ and $V_{z}=7.5 \mathrm{~km} / \mathrm{s}$, taken from Jaschek \& Valbousquet (1993). We assumed also a sky distribution with a cut- off in apparent magnitude at 6.5. We left two parameters free, namely the error of the radial velocity and the dispersion of the absolute magnitudes. The results are given in Tables 1 and 2. In Table 1 we have hold fixed the dispersion of the radial velocities and varied the dispersion of the absolute magnitudes, whereas in table 2 we have hold fixed the dispersion of the absolute magnitudes and varied the dispersion of the radial velocities.

In column five of Table 1 we have given the correction for the Malmquist bias, in the form of $-1.38 \sigma^{2}$. As can be seen by comparison with the next column, the real results deviate significantly after $\sigma=1.0$ from the formula. This is an interesting, but not unexpected result because it shows the limitations of the Malmquist formula as usually applied. It should be added that the latter has been derived using a hypothesis which does not apply to the distribution of intrinsically bright stars, namely the one of a constant space density.
Table 1. Results of numerical simulations carried out on 50 samples of 50 stars each. Assumed dispersion of radial velocities $= \pm 2 \mathrm{~km} / \mathrm{s}$

$\begin{array}{ccccc}\text { Disp. } M & M & & \text { Malmq. } & \text { Model } \\ 2.5 & -11.3 & 0.29 & 8.6 & 5.9 \\ 2.0 & -9.5 & 0.27 & 5.5 & 4.1 \\ 1.5 & -8.0 & 0.24 & 3.1 & 2.6 \\ 1.0 & -6.6 & 0.32 & 1.4 & 1.2 \\ 0.5 & -5.8 & 0.29 & 0.3 & 0.4 \\ 0.25 & -5.6 & 0.31 & 0.1 & 0.2\end{array}$

The first column gives the dispersion of the absolute magnitudes, the second the mean magnitude of the 50 samples, the third the dispersion of these, the fourth the Malmquist correction and the last the true correction, as taken from the model.

Table 2. Results of numerical simulations with 50 samples of 50 stars each $M=-5,6 \sigma(M)=0,5$ with different values of $\sigma(V)$

$\begin{array}{cccc}\text { disp. } V & M & \text { disp. } & \text { Malmquist corr. } \\ 0 & -5.9 & 0.21 & -5.5 \\ 5 & -5.9 & 0.22 & -5.5 \\ 10 & -5.9 & 0.24 & -5.5 \\ 15 & -5.8 & 0.25 & -5.4\end{array}$

The first column provides the radial velocity dispersion assumed, the second the mean of the 50 samples for the average absolute magnitude, the third the dispersion of these around the mean from the fifthy samples. The last column gives the values of $M$ with the Malmquist correction applied, according to the last column of Table 1.

As can be seen, even rather large dispersions of the radial velocity do not alter the results and from Tables 1 and 2 one can conclude that the galactic rotation method works well, in the sense that one recovers correctly the input values from samples of the order of 50 stars.

We can now deal with a second question, which is of similar importance, namely to assess with what precision the value of the galactic rotation constant can be obtained.

\subsection{Precision of the rotational constant $A$}

We have examined this question again by numerical simulation. What we have done is to assume the distances of the stars to be known and to determine how well an assumed input value of $A$ is found back in the samples. We fabricate samples with $M=-4$, and with a variable dispersion of $M$. We adopt for the galactic distribution a constant density in the galactic plane and an exponentially decreasing density outside, with $\beta=50 \mathrm{pc}$. For the velocity dispersions we use $15 \mathrm{~km} / \mathrm{s}$, and we use the standard solar motion derived previously $\left(V_{0}=20 \mathrm{~km} / \mathrm{s}, l_{0}=52\right.$, $\left.b_{0}=22\right)$ and assume that $A=15 \mathrm{~km} / \mathrm{s}$ and $K=0$. We have chosen three values for the dispersion of $M$, namely 
0.250 .50 and 0.75 . On the basis of these samples we determine $A$ and $K$. The results are given in Table $3 \mathrm{a}$. The table shows very convincingly that the error of $A$ is of the order of \pm 0.6 for samples of one thousand stars. For $K$ the situation is better but one needs at least samples of 400 stars to have dispersions of the order of $\pm 1 \mathrm{~km} / \mathrm{s}$. It is thus unjustified to quote the value of $K$ with one decimal point as it is often done, using samples of one hundred stars.

Table 3. Results of numerical simulations from 50 samples of 50 stars each a) Average absolute magnitude of the stars assumed known, $A=15 \mathrm{~km} / \mathrm{s.kpc} . K=0$

$\begin{array}{lllllll}\text { Dispersion in } M & 0.25 & & 0.50 & & 0.75 & \\ A & 16.1 & 2.8 & 16.2 & 2.8 & 16.6 & 3.6 \\ K & 0.1 & 2.7 & 0.1 & 3.1 & -0.4 & 2.8\end{array}$

b) For the same samples as above and using the same assumptions

$\begin{array}{lllllll}\text { Dispersion in } M & 0.25 & & 0.50 & & 0.75 & \\ (\overline{A . r}) & 17.0 & 6.8 & 16.6 & 6.7 & 16.3 & 7.1 \\ \bar{m} & 5.9 & 1.1 & 5.8 & 1.1 & 5.8 & 1.1 \\ \bar{M} & -4.37 & & -4.42 & & -4.38 & \end{array}$

Note: the first column under each dispersion gives the average of the 50 samples and the second column, the dispersion of the 50 samples around the mean.

Since the samples from which $A$ was determined in the literature (B-type stars, Cepheids) were certainly not larger than one thousand objects, the foregoing result implies that we do not know the value of $A$ to better than $\pm 1 \mathrm{~km} / \mathrm{s} . \mathrm{kpc}$. It may be argued that the finally adopted value of $A$ results from a comparison of different procedures- for instance from cluster distances and from supergiants. To this one can reply that a combination of the results of different methods contains always the danger of unexpected systematic errors.

As far as for example the use of supergiants goes, the value of $A$ derived from the large sample of supergiants by Humphreys (1970) depends strictly on the absolute magnitude system of Blaauw (1963) and a change in this system also implies a change in the value of $A$.

On the other hand, cluster distances depend on the splicing together of cluster sequences and this technique is based on a completely different set of assumptions, which may or may not be compatible with the results from supergiants. Let us enumerate briefly these assumptions. First, that from a given point on all cluster sequences are identical. This implies in short that different chemical compositions, binaries properties, rotations and magnetic fields have no influence on the position of the main sequence. Second, that interstellar reddening and extinction follows the same law in all clusters and associations. This excludes in particular circumstellar reddening and variable extinction in associations. Third, since only a limited number of nearby clusters exists, the result has rather large errors attached, from statistical grounds. For instance Hron (1987) finds, from a discussion of 105 young open clusters, $17.0 \pm 1.5 \mathrm{~km} / \mathrm{s} . \mathrm{kpc}$ (extreme values 14.3 and 18.3).

We shall assume in what follows that the value of $A$ is known to $\pm 1 \mathrm{~km} / \mathrm{s} . \mathrm{kpc}$

c) The average absolute magnitudes from the galactic rotation distances. Our next interest is to estimate the uncertainties of the average absolute magnitude one can obtain with the method of galactic rotation. We have thus calculated for the same samples the values of the product (A.r) and of the average apparent magnitude. These values are given in Table 3b.

From these values it is easy to deduce the average absolute magnitude by means of the formula:

$$
M=m+5-5 \cdot \log (A r / A)+C
$$

where $C$ is a correction term taking into account the fact that we are taking an average distance from a magnitude limited sample. Furthermore the absolute magnitude is not constant, but is represented by a Gaussian type luminosity function around $M_{0}$ with dispersion $\sigma$. Essentially this implies a Malmquist type correction on the absolute magnitude obtained from the method.

Since the galactic rotation method provides an "average" distance, it would seem preferable to derive a correction to the distance obtained, instead of applying a Malmquist type correction to the absolute magnitudes. But since both corrections are equivalent, the results obtained with one or the other procedure must coincide. The value of this correction for the distances can be evaluated with little difficulty. Be $r$ the average distance of the sample defined as:

$$
\bar{r}=\frac{\int_{0}^{\infty} r^{2} \cdot D(r) \varphi(M) r \cdot \mathrm{d} r}{\int_{0}^{\infty} r^{2} \cdot D(r) \varphi(M) \cdot \mathrm{d} r}
$$

where $\varphi(M)=C \cdot \exp \left(-\frac{\left(M-M_{0}\right)^{2}}{2 \sigma^{2}}\right)$ is the luminosity function assumed to be Gaussian $D(r)$ the density function, assumed to be constant. Call also:

$$
M_{0}=m+5+5 \cdot \log r_{0}
$$

Under these hypotheses it is easy to integrate and to show that:

$$
\log \bar{r}=\log r_{0}+\frac{7 \sigma^{2}}{50 \cdot \log e}
$$

Since $M-M_{0}=-5 .\left(\log r-\log r_{0}\right)$ one sees that the effect is similar to the Malmquist correction, which is also proportional to the square of the dispersion of the absolute magnitudes. It seems however pointless to go beyond this 
point, since the real distribution of stars in space does not obey a constant space density.

From our numerical simulation one sees that the average absolute magnitude comes out too bright by about 0.4 , independently of the value of the dispersion of the absolute magnitude. This is equivalent to an error of about $\pm 20 \%$ in the average distance.

Looking back on the first line of Table 3 one sees that also the values of A do not correspond exactly to what was introduced-one gets 16 instead of the $15 \mathrm{~km} / \mathrm{s}$ introduced.

In both cases one does not get back the values introduced. But what is more annoying is the size of the dispersions affecting these quantities-in the case of $A$ one has dispersions of the order of $20 \%$ and in the case of the product $(A r)$, of the order of $40 \%$. This implies that only very large samples can produce precise results.

\section{Application to real data}

\subsection{Galactic supergiants}

We have applied the method of the galactic rotation to a sample of galactic supergiants and brigth giants of luminosity classes Ia, Iab, Ib and II.

The sample was selected from the files of the SIMBAD database. It includes stars which fulfil the following conditions 1-to possess a radial velocity 2 -to possess an MK classification and 3-to possess an $U B V$ photometry or at least a $B V$ photometry. The final sample meeting all these conditions comprises more than 1200 stars of luminosity classes Ia, Ib, Ib and II. stars. This sample was then scrutinized to ensure its homogeneity. First of all we eliminated all stars with classifications which were not pure classes (for instance Ib-II ). Then we examined the position of the stars on the sky, to eliminate cluster members or double stars. To do so we rejected all stars lying within $0^{\prime \prime} 5$ of another member of the sample. The final sample was of more than 600 stars.

The next step was to derive the reddening. We used the intrinsic colors for each luminosity class as given by Schmidt-Kaler (1982) and dereddened each observed color pushing it back to the intrinsic color sequence on a line having a slope of 0.72 in the $U-B, B-V$ diagram. It should be added that there exists agreement in the literature on the intrinsic colors of supergiants, so that the choice of Schmidt-Kaler's values is not critical.

The value of the intrinsic color extrapolated "backwards" was then compared to the intrinsic color indicated by the spectral type. If the agreement was within $\pm 0^{\mathrm{m}} 16$, the reddening was judged to be acceptable. In a few cases however the two quantities differed by more than that amount. We have looked into the possibility of using a more complex de-reddening formula, including a color term, like:

$$
0.72+0.05 E(B-V)
$$

Using this formula we have recalculated the reddening for all stars. The differences are however rather small, so that the complex procedure was retained only for 19 stars with large reddening.

The distribution of the reddening for the different luminosity classes is given in Table 4 .

Table 4. Distribution of interstellar reddening in the stars of our sample

$\begin{array}{ccccc}E(B-V) & \text { II } & \text { Ib } & \text { Iab } & \text { Ia } \\ 0.0 .-0.1 & 190 & 94 & 77 & 35 \\ 0.1-0.2 & 72 & 60 & 35 & 26 \\ 0.2-0.3 & 44 & 56 & 24 & 19 \\ 0.3-0.4 & 38 & 51 & 21 & 18 \\ 0.4-0.5 & 36 & 56 & 22 & 25 \\ 0.5-0.6 & 35 & 25 & 19 & 28 \\ 0.6-0.7 & 21 & 21 & 16 & 22 \\ 0.7-0.8 & 14 & 22 & 11 & 18 \\ 0.8-0.9 & 6 & 23 & 5 & 12 \\ 0.9-1.0 & 4 & 11 & 9 & 13 \\ 1.0-1.1 & 4 & 7 & 5 & 5 \\ 1.1-1.2 & 1 & 2 & 2 & 9 \\ 1.2-1.3 & & 3 & 1 & 4 \\ 1.3-1.4 & & 1 & & 2 \\ 1.4-1.5 & & 1 & & \end{array}$

Each case gives the number of stars of a certain luminosity class having a certain amount of reddening.

It can be seen that very large reddenings-larger than one magnitude-are exceptional. We use now the values the kinematic parameters, obtained in our previous paper (Jaschek et al. 1995) from a sample of B-type stars to calculate the average absolute magnitudes of the supergiants, based upon a sample of 615 stars. In this sample we have excluded nearby stars because for these the galactic rotation effect is small and because they contain also the kinematic effects of the local system The distances of the stars in the sample lie between 400 and 2000 pc. The use of the parameters for B-type stars is justified in that both the B-type stars and the supergiants are young stars, so that presumably their kinematics is also very similar.

Specifically we have used the following parameters $V_{0}=17.4 \mathrm{~km} / \mathrm{s} l_{0}=60.7 b_{0}=20.9 K=-1.2 \mathrm{~km} / \mathrm{s}$ $A=15.5 \mathrm{~km} / \mathrm{s} . \mathrm{kpc}$. The resulting absolute magnitudes are given in Table 5 .

A first glance at the table shows very large values for the dispersions (see Col. 4). This implies either that the method is incapable of deriving precise results, which is in contradiction with the results from Sect. 1. The other explanation is that the supergiant sequences do not exist. This means that these objects cover the whole upper part of the HR diagram.

In favor of the second explanation we have also the fact that many spectral classifications of supergiants use intermediate luminosity classes like Ib-II or Iab-Ib. From 
Table 5. Absolute magnitudes of supergiants

$\begin{array}{lcccc}\text { Lumin.group } & \text { Number } & M & D & \text { Malm } \\ \text { Ia } & 50 & -8.0 & 1.37 & -7.6 \\ \text { Iab } & 88 & -6.7 & 2.81 & -6.3 \\ \text { Ib } & 208 & -5.7 & 2.02 & -5.3 \\ \text { II } & 256 & -3.5 & 2.25 & -3.1\end{array}$

$M$ is the average absolute magnitude

$D$ is the dispersion of the $\mathrm{M}$ values around the mean

Malm= stands for column three with the Malmquist correction (see text) applied.

our original sample of 1200 supergiants, half were eliminated because we retained only those objects classified as "pure" classes (like Ib or II). Such a large proportion of intermediates does not occur neither for dwarfs nor for giants. Notice also that up to now no calibration of these intermediate luminosity classes has been made. Another independent fact which goes in the same direction (but shall not be discussed here) is that in purely photometric diagrams in which the supergiants observed in a cluster are plotted in an $M v$ vs. color diagram, no clear sequences have ever been detected in the upper part of the HR diagram. We retain thus as the most likely explanation for the large dispersion the fact that no real sequences do exist like for giants or dwarfs.

We must next apply the Malmquist correction for the average absolute magnitudes found, since our sample is limited in apparent magnitude For this we need a correct value of the dispersion. We could use the value derived from the sample itself, but in such case we include with full weigth the errors of spectral classification. Since we are grouping all stars of a given luminosity class together, an error in spectral class has no influence, but that of luminosity class has a very large influence. An error of one luminosity class- for instance from Iab to Ib- implies an error of more than one magnitude, which is larger than (or at least comparable to) the size of the Malmquist correction. It seems that it is better to assume the dispersion of $M$ to be equal to half the distance between the values found for the different groups. The result is that sigma lies in the interval 0.5 to 0.6 for the first three groups, which lead to Malmquist corrections of the order of 0.4 . The values corrected by this amount are given in the fifth column of Table 5. We shall adopt the same value for class II stars.

\subsection{Comparison with previous calibrations}

One can compare these values with those tabulated by Blaauw and by Schmidt-Kaler. The comparison is given in Table 6. Since both authors provide absolute magnitudes as a function of luminosity class and spectral type, we must fabricate from their values averages over the spectral type. We have obtained the average values for both authors in two different ways- in the first case by averaging the absolute magnitudes values for B0, A0, F0...M0 and in the second case by averaging B5, A5...M5. As can be seen for classes Ia, Iab and Ib the agreement is excellent. It is somewhat worse for the class II objects due to the large range of variations over spectral type.

Table 6. Comparison of results

$\begin{array}{lccccc}\text { Lum.class } & \text { T.P. } & \mathrm{SK}(1) & \mathrm{Bl}(1) & \mathrm{SK}(2) & \mathrm{Bl}(2) \\ \mathrm{Ia} & -7.6 & -7.45 & -7.5 & -7.4 & -7.65 \\ \mathrm{Iab} & -6.3 & -6.2 & -6.2 & -6.2 & -6.2 \\ \mathrm{Ib} & -5.3 & -5.0 & -4.9 & -4.9 & -4.8 \\ \mathrm{II} & -3.1 & -3.0 & -3.0 & -2.7 & -2.6\end{array}$

Note: T.S. $=$ this paper SK = Schmidt-Kaler Bl = Blaauw

(1) refers to averaging absolute magnitudes at B0, A0...M0 and

(2) to averaging at B5, A5...M5.

The agreement with our values is good, confirming in an independent way the correctness of the scale. Our values have the advantage of being derived from a large sample of stars, using one clearly defined method. This is not the case with either Blaauw's or Schmidt-Kaler's values, for which one lacks details how the calibration was done. The data for the stars used in the sample are available from the CDS.

The drawback of our method is that we are unable to provide the variation with spectral type within a given luminosity class. Several attempts in this sense have failed. This is due to the fact that our method needs large samples, which are unavailable at present We can explain this with the help of a small statistics. The method of galactic rotation distances is based upon the use of radial velocities. If one calculates the dispersion of the radial velocities, such as they were observed, one finds $25.6 \mathrm{~km} / \mathrm{s}$. After correcting for the solar motion, one finds 21.5., i.e. a reduction of the dispersion by five $\mathrm{km} / \mathrm{s}$. The application of galactic rotation reduces this dispersion to 20.2 , implying that the galactic rotation is a small effect - in any case much smaller than the effect of the solar velocity. Since all our conclusions are based upon this small effect, it is clear that subsets have to be very large to render useful results.

The smallness of the galactic rotation term explains a curious fact. We have used in the preceding calculations the simple galactic rotation formula, which is valid within a small heliocentric distance. ( $<1 \mathrm{kpc})$ Since some of our supergiants have distances of up to two kpc, we have used more complete formulae for the galactic rotation, namely the one used by Comerón et al. (1994) and the one given by Smart (1938), which uses quadratic terms in the heliocentric distance. To our surprise we have found that the more complete formulae do not produce a decrease of the dispersion of the radial velocities, as could be expected. The formula using 
quadratic terms in the heliocentric distance presents furthermore the unavoidable difficulty that an inversion of it, for the obtention of the heliocentric distance presents two roots. Since no obvious choice for either one can be made a priori, this formula is useless for the method of galactic rotation distances.

As a last point we shall try to see if the solution found for the absolute magnitudes is stable. In the broad sense this means that small variations in the input parameters produce (only) small changes in the absolute magnitudes. This is a rather crucial point, given the smallness of the galactic rotation effect as shown above.

Since there exist five kinematic parameters which have to be fixed $\left(V_{0}, l_{0}, b_{0}, K, A\right)$ strictly one would have to study how the absolute magnitudes varies with each of these five parameters. In practice however $l_{0}$ and $b_{0}$ seem to have little influence and the same can be said for $K$, so that we have examined in more detail the variations of $M$ produced by a variation of $V_{0}$ and $A$, using the data from our sample. The results are given in Table 7 .

Table 7. Variations of the absolute magnitude with different values of $V_{0}$ and $A$

$\begin{array}{lccccc}\text { Parameters } & & M(\mathrm{Ia}) & M(\mathrm{Iab}) & M(\mathrm{Ib}) & M(\mathrm{II}) \\ A=14 & V=17 & -7.0 & -5.7^{*} & -4.7 & -2.4^{*} \\ A=14 & 18 & -7.0 & -5.7 & -4.7 & -2.5 \\ A=14 & 19 & -7.0^{*} & -5.4 & -4.8^{*} & -2.6 \\ A=15 & V=17 & -6.8 & -5.5^{*} & -4.6 & -2.3^{*} \\ A=15 & 18 & -6.8 & -5.6 & -4.6 & -2.3 \\ A=15 & 19 & -6.8^{*} & -5.2 & -4.6^{*} & -2.3 \\ A=16 & V=17 & -6.7 & -5.4^{*} & -4.4 & -2.2^{*} \\ A=16 & 18 & -6.7 & -5.4 & -4.5 & -2.2 \\ A=16 & 19 & -6.7 & -5.1 & -4.5 & -2.2\end{array}$

Are signaled with an asterisk in each column those combinations of parameters which produce the least dispersion of the results. If two values are marked, the dispersions are equal.

An examination of Table 7 reveals the nice fact that small variations in $V$ and $A$ produce small variations in the absolute magnitude.In class Ia the extreme variation is 0.3 , in class Iab 0.6, in class Ib 0.4 and in class II 0.4 . In summary, the solution seems to be stable.

\section{Summary}

In this paper we have obtained five results:

1) The method of galactic rotation distances is shown to work well on the basis of numerical experiments.

2) The average absolute magnitude of stars of luminosity classes Ia, Iab, Ib and II is derived from a large set of stars, not available before, using one single well defined method. The results obtained confirm the general trend of the Blaauw and Schmidt-Kalers calibration.

3) no finer subdivisions with spectral type can be established due to the lack of sufficiently large samples.

4) The dispersion of the average absolute magnitudes is very large. We interpret this as implying that no real supergiant sequences do exist, but that supergiants cover more or less uniformly a region of the HR diagram.

5) We have examined the dependence of the absolute magnitudes upon small variations of $V$ and $A$ and found that the dependence is not very strong, so that the solution seems to be stable.

In addition the value of the Malmquist correction for mean distances is derived.

Acknowledgements. We wish to thank the referee Dr. F. Comerión for helpful comments.

\section{References}

Blaauw A., 1963, "Basic Astronomical Data", Vol. 3. In: Strand K.A. (ed.). Univ. Chicago Press

Comerón F., Torra J., Gómez A.E., 1994, A\&A 286, 789

Hron J., 1987, A\&A 176, 34

Humphreys R., 1970, AJ 75, 602

Kuijken K, Tremaine S., 1992, "Dynamics of disc galaxies", Varberg Castle Conf., Sweden. In: Sundelins B. (ed.), p. 71

Jaschek C., Valbousquet A., 1993, A\&A 275, 472

Jaschek C., Valbousquet A., Luri X., 1994, A\&A 291, 439

Jaschek C., Valbousquet A., Ochsenbein F., 1995, A\&A (accepted)

Schmidt-Kaler Th., 1982, in Landolt-Bornstein. group 6, Vol. 2b. Springer Verlag, Berlin, p. 373

Smart W.M., 1938, "Stellar Dynamics". Cambridge University Press, p. 374 